\title{
A Network-Coded Diversity Protocol for Collision Recovery in Slotted ALOHA
}

\section{Networks}

\author{
G. Cocco*, N. Alagha ${ }^{\Uparrow}$, C. Ibars* and S. Cioni $₫$ \\ ${ }^{*}$ Centre Tecnològic de Telecomunicacions de Catalunya - CTTC \\ Parc Mediterrani de la Tecnologia, Av. Carl Friedrich Gauss 7 08860, \\ Castelldefels - Spain \\ 『European Space Agency - ESTEC, Noordwijk - The Netherlands \\ giuseppe.cocco@cttc.es, nader.alagha@esa.int, christian.ibars@cttc.es, \\ stefano.cioni@esa.int
}

\begin{abstract}
We propose a collision recovery scheme for symbol-synchronous slotted ALOHA (SA) based on physical layer network coding over extended Galois Fields. Information is extracted from colliding bursts allowing to achieve higher maximum throughput with respect to previously proposed collision recovery schemes. An energy analysis is also performed, and it is shown that, by adjusting the transmission probability, high energy efficiency can be achieved. The paper also addresses several practical aspects, namely frequency, phase, and amplitude estimation, as well as partial symbol asynchronism. A performance evaluation is carried out using the proposed algorithms, revealing remarkable performance in terms of normalized throughput.
\end{abstract}

This work was partially supported by the European Commission under project ICT-FP7-258512 (EXALTED), by the Spanish Government under project TEC2010-17816 (JUNTOS) and project TEC2010-21100 (SOFOCLES), and by Generalitat de Catalunya under grant 2009-SGR-940. ${ }^{\dagger}$ G. Cocco is partially supported by the European Space Agency under the Networking/Partnering Initiative. Part of the present work will be presented at the 6th Advanced Satellite Multimedia Systems Conference (ASMS), to be held in Baiona (Spain) in 2012. This work has been submitted to IEEE Journal on Selected Areas in Communications. 


\section{Index Terms}

Physical-layer network coding; slotted ALOHA; random access networks; collision recovery; satellite communications; CRDSA; energy efficient multiple access; spectral efficient multiple access.

\section{INTRODUCTION}

The throughput of Slotted ALOHA (SA) systems is limited by the collisions that take place when more than one node accesses the channel in the same time slot. This limitation is particularly problematic in satellite networks with random access, where the long round-trip time (RTT) greatly limits feedback from the receiver, for example to perform load control or to request retransmission.. Techniques like Diversity Slotted ALOHA (DSA) [1], in which each packet is transmitted more than once, have been proposed in order to increase the probability of successful detection. The spectral efficiency of SA systems can be increased by exploiting the collided signals. In Contention Resolution Diversity Slotted ALOHA (CRDSA) [2] the collided signals are exploited using an iterative interference cancelation (IC) process. In CRDSA each packet is transmitted more than once and uncollided packets are subtracted from slots in which their replicas are present. In [3] a packet-level forward error correction (FEC) code has been applied to CRDSA, while in [4] a convergence analysis and optimization of CRDSA has been proposed.

Another technique that allows to extract information from colliding signals is physical layer network coding (PHY NC). PHY NC was originally proposed to increase spectral efficiency in two-way relay communication [5] by having the relay decoding the collision of two signals under the hypothesis of symbol, frequency and phase synchronism. Several studies have been reported in the literature about synchronization issues, gain analysis and ad-hoc modulation techniques for PHY NC in the case of two colliding signals [6][7][8]. In [9] PHY NC has been applied in the satellite context for pairwise node communication. In [10] and [11] it has been proposed to apply PHY NC to determine the identity of transmitting nodes in case of ACK collision in multicast networks by using energy detection and ad-hoc coding schemes, under the hypothesis of phase synchronous signal superposition at the receiver. In [12] the decoding of multiple colliding signals over generally complex channels has been studied from an information theoretical point of view. In [13] PHY NC has been applied for collision resolution in ALOHA systems with feedback from the receiver, under the assumption of frequency synchronous transmitters.

In this paper we present a new scheme named Network-Coded Diversity Protocol (NCDP), that leverages on PHY NC in extended Galois Fields for recovering collisions in symbol-synchronous SA systems. Once the PHY NC is applied to decode the collided bursts, the receiver uses common matrix manipulation techniques over finite fields to recover the original messages, which results in a 
high-throughput scheme. The proposed scheme and analysis differ from previous works on collision resolutions at both system (SYS) level and physical (PHY) level:

SYS: - Unlike in [13], we assume that transmissions are organized in frames. We consider two different setups. In one, the nodes do not receive any feedback from the receiver. If on the one hand the absence of feedback leads to a best-effort scheme, in which there is no guarantee for a message to be received, on the other hand it notably simplifies the system architecture and decreases the total amount of energy spent per received packet. In the second setup that we consider, instead, feedback is allowed from the receiver. In particular, we consider an automatic repeat request (ARQ) scheme, in which a node receives an acknowledgement (ACK) or a negative acknowledgement (NACK) from the receiver in case a message is or is not correctly received, respectively. A message for which a NACK has been received is retransmitted in a different frame. The retransmission process goes on until the message is acknowledged.

- We evaluate jointly the spectral efficiency (average number of messages successfully received per slot) and the energy consumption (average amount of energy needed for a message to be correctly received) of the proposed scheme and compare it with other collision resolution schemes previously proposed in the literature.

PHY: - We use extended Galois Fields, i.e., $G F\left(2^{n}\right)$ with $n>2$, instead of $G F(2)$, which is generally used in PHY NC. This allows to better exploit the diversity of the system, leading to increased spectral efficiency and, depending on the system load, to an increased energy efficiency.

- We take into account frequency and phase offsets at the transmitters when applying PHY $\mathrm{NC}$ for an arbitrary number of colliding signals. Up to our knowledge, the issue of frequency offsets in PHY NC has been previously addressed only for the case of two colliding signals. See, e.g., [14], [15] and references therein.

- We show the feasibility of channel estimation for PHY NC in the presence of more than two colliding signals, unlike previous works where only two colliding signals were considered (see, e.g., [16]).

- We study the effect of non perfect symbol synchronism on the decoder FER for an arbitrary number of colliding signals and propose four different methods to compensate for such effect.

The rest of the paper is organized as follows. In Section II we present the system model. Section III describes how the channel decoding works in case of a generic number of colliding signals with independent frequency and phase offsets. In Section IV the proposed scheme is described, while its performance is studied in Section $\mathrm{V}$ in terms of both spectral and energy efficiency. Section $\mathrm{VI}$ deals 
with issues such as channel estimation and error detection, which are fundamental for a practical implementation of the proposed scheme. Section VII is dedicated to the effect of imperfect symbol synchronization on the decoder performance in case of multiple colliding signals, and different schemes to overcome such effects are presented. In Section VIII we present the numerical results, while Section IX contains the conclusions.

\section{System Model}

Let us consider the return link (i.e, the link from a user terminal to the satellite/base station) of a multiple access system with $M$ transmitting terminals, $T_{1}, \ldots \ldots, T_{M}$, and one receiver $R$. Packet arrivals at each transmitter are modeled as a Poisson process with rate $\frac{G}{M}$, which is independent from one transmitter to the other. Each packet $\mathbf{u}_{i}=\left[u_{i}(1), \ldots, u_{i}(K)\right]$ consists of $K$ binary symbols of information $u_{i}(\xi) \in\{0,1\}$, for $\xi=1, \ldots, K$. We assume that, upon receiving a message, each terminal $T_{i}$ uses the same linear channel code of fixed rate $r=\frac{K}{N}$ to protect its message $\mathbf{u}_{i}$, obtaining the codeword $\mathbf{x}_{i}=\left[x_{i}(1), \ldots, x_{i}(N)\right]$, where $x_{i}(l) \in\{0,1\}$ for $l=1, \ldots, N$. For ease of exposition a BPSK modulation is considered. Each codeword $\mathbf{x}_{i}$ is BPSK modulated (using the mapping $0 \rightarrow-1$, $1 \rightarrow+1)$, thus obtaining the transmitted signal

$$
s_{i}(t)=\sum_{l=1}^{N} b_{i}(l) g\left(t-l T_{s}\right),
$$

where $T_{s}$ is the symbol period, $b_{i}(l)$ is the BPSK mapping of $x_{i}(l)$ and $g(t)$ is the square root raised cosine (SRRC) pulse. The signal $s_{i}(t)$ is called burst.

In the following we will refer to a time division multiple access (TDMA) scheme. However, the techniques proposed in the following can be also applied to other access schemes, such as multifrequency-TDMA (MF-TDMA), in which a frame may include several carriers, or code division multiple access (CDMA), where NCDP can be used to recover collisions in each of the code sub-channels. It should be noted that the proposed technique still relies on single carrier transmission of each user terminal. From the user terminal perspective no significant change is required. Transmissions are organized in frames. Each frame is divided into $S$ time slots. The number $S$ of time slots that compose a frame is constant, i.e., it does not change from one frame to the other. The duration of each slot is equal to about $N$ burst symbols. When more than one burst is transmitted in the same slot a collision occurs at the receiver. A collision involving $k$ transmitters is said to have size $k$. We assume symbolsynchronous transmissions, i.e., in case of a collision, the signals from the transmitters add up with symbol synchronism at $R$. The received signal before matched filtering and sampling at $R$, in case of a collision of size $k$ (assuming, without loss of generality, the first $k$ terminals collide), is:

$$
y(t)=h_{1}(t) s_{1}(t)+\ldots+h_{k}(t) s_{k}(t)+w(t)
$$


where $s_{i}(t)$ is the burst transmitted by user $i, w(t)$ is a complex additive white Gaussian noise (AWGN) process while $h_{i}(t)$ takes into account the channel from terminal $i$ to the receiver. $h_{i}(t)$ can be expressed as:

$$
h_{i}(t)=A_{i} e^{j\left(2 \pi \Delta \nu_{i} t+\varphi_{i}\right)},
$$

where $A_{i}=\left|h_{i}\right|$ is a lognormally distributed random variable modeling the channel amplitude of transmitter $i$, while $\Delta \nu_{i}$ and $\varphi_{i}$ are the frequency and phase offsets with respect to the local oscillator in $R$, respectively. We assume that the amplitude $A_{i}$ and the frequency offset $\Delta \nu_{i}$ remain constant within one frame [2] while $\varphi_{i}$ is a random variable uniformly distributed in $[-\pi,+\pi]$ that changes independently from one slot to the other. The fact that $\varphi_{i}$ changes from one slot to the other is due to the phase noise at the transmitting terminals [2]. Assuming that the frequency offset is small compared to the symbol rate $1 / T_{s}\left(\Delta \nu T_{s} \ll 1\right)$, the sample taken at time $t_{l}$ after matched filtering of signal $y(t)$ is:

$$
r\left(t_{l}\right)=h_{1}\left(t_{l}\right) q_{1}\left(t_{l}\right)+\ldots+h_{k}\left(t_{l}\right) q_{k}\left(t_{l}\right)+n\left(t_{l}\right),
$$

where $q(t)=s(t) \oplus g(-t)$, while $n\left(t_{l}\right)$ 's are i.i.d. zero mean complex Gaussian random variables with variance $N_{0}$ in each component. Note that even in case a BPSK modulation is used, as we are assuming in this paper, both the I and Q components of the received signal are considered by the receiver. This is because the phases of the users have random relative offsets and thus both components carry information relative to the useful signal. The random relative offsets must be taken into account by the decoder, as they cannot be eliminated by the demodulator. We consider this more in detail in Section [II]

We assume that the receiver has knowledge of the nodes that are transmitting, as well as the full channel state information at each time slot. As we are considering a random access scheme, the knowledge about nodes identity cannot be available a priori at the receiver. Instead, nodes identity must be determined by $R$ starting from the received signal, even in case a collision occurs. This can be achieved by having the transmitting nodes adding an orthogonal preamble in each transmitted burst, assuming that the probability that two nodes use the same preamble is negligible [2]. We discuss the issue of node identification and channel estimation more in detail in Section VI

\section{Multi-User Physical Layer Network Coding}

In this section we describe the way the received signal is processed by the receiver $R$ in case of a collision

When a collision of size $k$ occurs, i.e., $k$ bursts collide in the same slot, the receiver tries to decode the bit-wise XOR of the $k$ transmitted messages. This can be done by feeding the decoder with the log-likelihood ratios (LLR) for the received signal. The calculation of the LLRs for a collision of generic 
size $k$ in case of BPSK modulation was presented in [13]. In the following we include the effect of frequency offset in the calculation of the LLRs, which was not taken into account in [13].

When signals from $k$ transmitters collide, the received signal at $R$ is given by (2). Each codeword $\mathbf{x}_{i}$ is calculated from $\mathbf{u}_{i}$ as $\mathbf{x}_{i}=\mathcal{C}\left(\mathbf{u}_{i}\right)$, where $\mathcal{C}($.$) is the channel encoder operator. All nodes use the same$ linear code $\mathcal{C}($.$) . Starting from r(t)$, the receiver $R$ wants to decode codeword $\mathbf{x}_{s} \triangleq \mathbf{x}_{1} \oplus \mathbf{x}_{2} \oplus \ldots \oplus \mathbf{x}_{k}$, where $\oplus$ denotes the bit-wise XOR. In order to do this the decoder of $R$ is fed with vector $\mathbf{L}^{\oplus}=$ $\left[L^{\oplus}(1), \ldots, L^{\oplus}(N)\right]$ of LLRs for $\mathbf{x}_{s}$, where:

$$
L^{\oplus}(l)=\ln \left\{\frac{\sum_{i=1}^{\left\lfloor\frac{k+1}{2}\right\rfloor} \sum_{m=1}^{\left(\begin{array}{c}
k \\
2 i-1
\end{array}\right)} e^{-\frac{\left|r\left(t_{l}\right)-\mathbf{d}^{o}(2 i-1, m)^{T} \mathbf{h}\left(t_{l}\right)\right|^{2}}{2 N_{0}}}}{\sum_{i=1}^{\left\lfloor\frac{k+1}{2}\right\rfloor} \sum_{m=1}^{\left(\begin{array}{c}
k \\
2 i
\end{array}\right)} e^{-\frac{\left|r\left(t_{l}\right)-\mathbf{d}^{e}(2 i, m)^{T} \mathbf{h}\left(t_{l}\right)\right|^{2}}{2 N_{0}}}}\right\},
$$

$\mathbf{h}\left(t_{l}\right)$ being a column vector containing the channel coefficients of the $k$ transmitters at time $t_{l}$ (which change at each sample due to frequency offsets), while $\mathbf{d}^{o}(2 i-1, m)$ and $\mathbf{d}^{e}(2 i, m)$ are column vectors

containing one (the m-th) of the $\left(\begin{array}{c}k \\ 2 i-1\end{array}\right)$ or $\left(\begin{array}{c}k \\ 2 i\end{array}\right)$ possible permutations over $k$ symbols (without repetitions) of an odd or even number of symbols with value “ +1 ”, respectively. Equation (5) is derived considering that an even or an odd number of symbols with value +1 adding up at $R$ must be interpreted by the decoder as a 0 or a 1 , respectively. The derivation of $L^{\oplus}(l)$ is detailed in the Appendix (see [6] and [8] for an extension to higher order modulations). If the decoding process is successful, $R$ obtains the message $\mathbf{u}_{s} \triangleq \mathbf{u}_{1} \oplus \ldots \oplus \mathbf{u}_{k}$. In Section VI the FER curves for different collision sizes obtained using these LLR values are shown.

\section{Network Coded Diversity Protocol}

In this section we present our network-coded diversity protocol (NCDP) which aims at increasing the throughput and reducing packet losses in Slotted ALOHA multiple access systems. In the first part of the section we recall some basics of finite field arithmetics, while in the second part we describe the NCDP at the transmitter and at the receiver side.

\section{A. Basics of Finite Fields}

A finite field is a closed set with respect to sum and multiplication with finitely many elements. Finite fields are often denoted as $G F\left(s^{n}\right)$, where $s$ is a prime number, $n$ is a positive integer and $G F$ stands for Galois Field. If $n=1$ all operations (sum, subtraction, multiplication and division) in the field coincide with operations over natural numbers modulo $s$. If $n>1$ the field is said to be an extended Galois Field (EGF). In an EGF each element can be represented as a polynomial of degree lower than $n$ and coefficients in $G F(s)$. An element in an EGF can be represented using the coefficients of the corresponding polynomial representation. Thus, a string of $n$ bits can be interpreted as an element in $G F\left(2^{n}\right)$. Along the same line, a string of $N=n \cdot L$ bits, $L \in \mathcal{N}$, can be represented as a vector in an $L$-dimensional space over $G F\left(2^{n}\right)$ (see [17] for more details). 
The sum operation in an EGF is done coefficient-wise. The sum of two elements in $G F\left(2^{n}\right)$ can be calculated as the bit-wise XOR of the two $n$-bits strings corresponding to the two elements to add.

The product in an EGF can be calculated through polynomial multiplication modulo an irreducible polynomial which characterizes the field. Subtraction and division are defined as the inverse operations of sum and product, respectively, and calculated accordingly.

Finally, let us consider a system of linear equations in $G F\left(2^{n}\right)$ with $N^{t x}$ variables and $S$ equations, $S \geq N^{t x}$, with an associated $S \times N^{t x}$ coefficient matrix A having elements in $G F\left(2^{n}\right)$. The system admits a unique solution iff the associated coefficient matrix $\mathbf{A}$ has exactly $N^{t x}$ linearly independent columns (rows).

\section{B. NCDP: Transmitter Side}

Assume that node $i$ has a message $\mathbf{u}_{i}$ to deliver to $R$ during frame $f$. We call active terminals the nodes that have packets to transmit in a given frame. Each message is transmitted more than once within a frame, i.e., several replicas of the same message are transmitted. We will give details about the number of replicas transmitted within a frame in next section. Before each transmission, node $i$ pre-encodes $\mathbf{u}_{i}$ as depicted in Fig. 1. The pre-coding process works as follows. $\mathbf{u}_{i}$ is divided into $L=\frac{K}{n}$ blocks of $n$

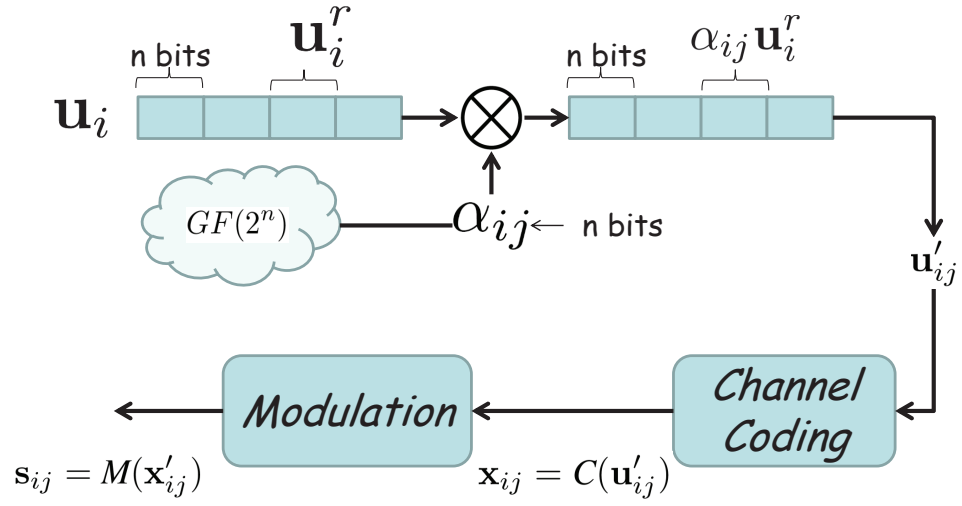

Fig. 1. NCDP pre-encoding, channel coding and modulation scheme at the transmitter side. The message to be transmitted is divided into sub-blocks. Each sub-block is multiplied by a coefficient $\alpha_{i j} \in G F\left(2^{n}\right)$. Coefficients $\alpha_{i j}, j \in\{1, \ldots, S\}$ are chosen at random in each time slot. After the multiplication, the message is channel-encoded, a header is attached and the modulation takes place.

bits each. At each transmission a different coefficient $\alpha_{i j}, j \in\{1, \ldots, S\}$, is drown randomly according to a uniform distribution in $G F\left(2^{n}\right)$. If $\alpha_{i j}=0$, terminal $T_{i}$ does not transmit in slot $j$. Each of the $L$ blocks $\mathbf{u}_{i}^{r}, r \in\{1, \ldots, L\}$, is interpreted as an element in $G F\left(2^{n}\right)$ and multiplied by $\alpha_{i j}$. We call $\mathbf{u}_{i j}^{\prime}$ the message $\mathbf{u}_{i}$ after the multiplication by $\alpha_{i j} . \mathbf{u}_{i j}^{\prime}$ is then channel encoded, generating the codeword $\mathbf{x}_{i j}=C\left(\mathbf{u}_{i j}^{\prime}\right)$. After channel coding, a header $p_{i}$ is added to $\mathbf{x}_{i j}$. Such header is chosen within a set of 
orthogonal codeword (e.g. Walsh-Hadamard). The same header $p_{i}$ is used for all transmissions of node $i$ within frame $f$, i.e., it does not change within a frame. Once the header is attached, $\mathbf{x}_{i j}$ is BPSK modulated and transmitted.

The choice of the coefficients and of the header is done as follows. Node $i$ draws a random number $\mu . \mu$ is used to feed a pseudo-random number generator, which is the same for all terminals and is known at $R$. The first $S$ outputs of the generator are used as coefficients. The header is uniquely determined by $\mu$, i.e, there is a one-to-one correspondence between the set of values that can be assumed by $\mu$ and the set of available orthogonal headers. The orthogonality of the preambles allows the receiver to know which of the active terminals in frame $f$ is transmitting in each time slot. Moreover, as the header univocally determines $\mu$ and thus the set of coefficients used by each node, $R$ is able to know which coefficient is used by each transmitter in each slot. As we we will see in Section IV-C this is of fundamental importance for the decoding process. As said before, the set of headers is a set of orthogonal words, such as those usually adopted in CDMA. The fundamental difference with respect to a CDMA system is that in such system the orthogonality of the codes is used to orthogonalize the channels and expand the spectrum, while in NCDP the orthogonality of the preamble is used only for determining the identity of the transmitting node, which is obtained without any spectral expansion, as the symbol rate $1 / T_{s}$ is equal to the chip rate (i.e., the rate at which the modulated symbols are transmitted over the channel) [2].

\section{NCDP: Receiver Side}

The decoding scheme at the receiver side is illustrated with an example in Fig. 2 and Fig. 3. In the example, a frame with $S=4$ slots and $N^{t x}=3$ active terminals are considered. In each slot the receiver uses the orthogonal preamble of each burst to determine which node is transmitting and which coefficient has been used for that burst. As described in Section IV-B the coefficients used by a node in each burst are univocally determined by the preamble. The preamble can be determined at $R$ using a bank of correlators which calculates in parallel the correlation of the received signal with each element in the set of available preambles. The preamble is also used by $R$ to estimate the channel for each of the transmitters. The details about the channel estimation are given in Section VI-A. Once the channel has been estimated, the decoder applies PHY NC to calculate the bitwise XOR of transmitted messages, as detailed in Section [II]. The receiver tries to channel-decode the received signals using PHY NC. According to what is stated in Section IV-A and Section IV-B, the bitwise XOR is interpreted as a sum in $G F\left(2^{n}\right)$. Thus the slots that have been correctly decoded are interpreted as a system of equations in $G F\left(2^{n}\right)$ with coefficients $\alpha_{i j}$, which are known to the receiver through the headers (see Fig. 2). At this point, if the coefficient matrix $\mathbf{A}$ has full rank, $R$ can recover all the original messages using common matrix manipulation techniques in $G F\left(2^{n}\right)$ (see Fig. 3). If $\mathbf{A}$ is not full rank, not all 


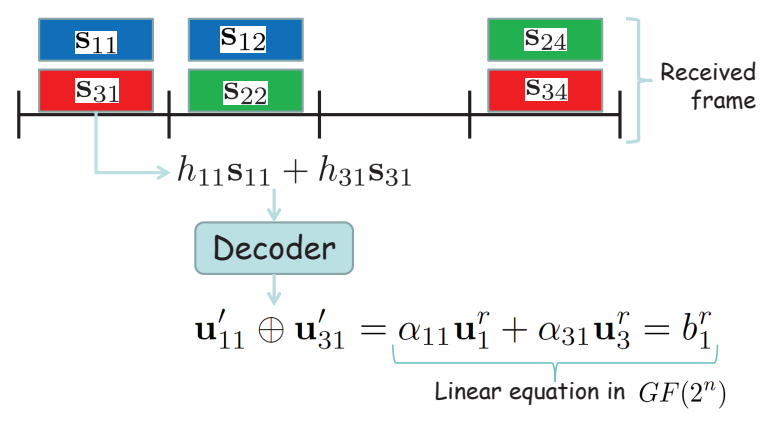

Fig. 2. For each of the slots the receiver uses the orthogonal preambles to determine the which node is transmitting. With the same preamble the channel from each of the transmitters in the slot to $R$ is estimated. The channel $h_{i j}, j \in\{1, \ldots, S\}$ changes at each slot due to phase noise, according to the channel model described in Section [II Once the channel has been estimated, the decoder applies MU PHY NC to calculate the bitwise XOR of transmitted messages. The bitwise XOR corresponds to a linear equation in $G F\left(2^{n}\right)$ with coefficients $\alpha_{i j}$ which are known to the receiver through the header. In the figure only bursts with non-zero coefficients are shown.

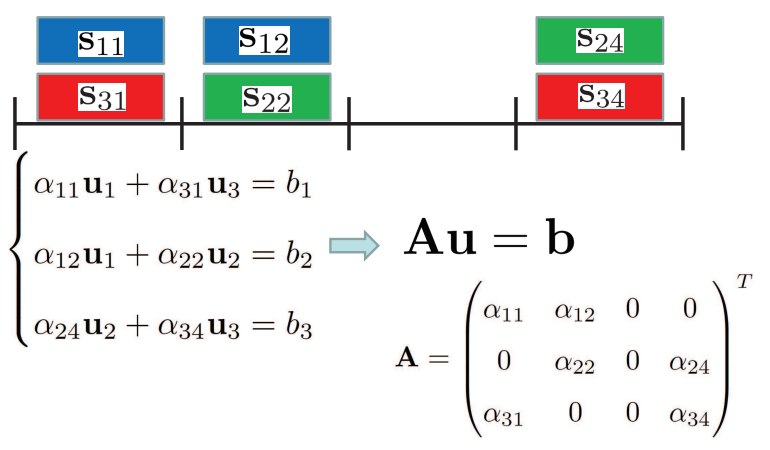

Fig. 3. The receiver tries to channel-decode all of the occupied slots, thus obtaining a system of equations in $G F\left(2^{n}\right)$. At this point, if the matrix $\mathbf{A}$ of coefficients is full rank, $R$ can obtain all the original messages. If $\mathbf{A}$ is not invertible, $R$ can decode the "clean" bursts (i.e., the bursts that did not experience collision), then subtract them from the slots where their replicas are. The procedure goes on until there are no more clean bursts. In the figure, ${ }^{T}$ represents the transpose operator.

the transmitted packets can be recovered. However, a part of them can still be retrieved using matrix manipulation techniques such as Gaussian elimination. The decoding process in case of rank deficient coefficient matrix is analyzed in Section $\mathrm{V}$

\section{Throughrut AND ENERGY ANALYSIS}

During each frame users buffer packets to be transmitted in the following frame. Each node transmits its packet more than once within a frame, randomly choosing a new coefficient in $G F\left(2^{n}\right)$ independently at each transmission. As described in the previous section, the coefficients can be generated using a pseudo-random number generator fed with a seed which is univocally determined by the chosen orthogonal preamble. Using the preamble the receiver can build up a coefficient matrix $\mathbf{A}$ for each frame, with $\mathbf{A}_{i, j}=\alpha_{i j}, \alpha_{i j} \in\left\{1, \ldots, 2^{n}-1\right\}$, such as the one represented in Table [ Columns represent time slots while rows represent the active terminals, i.e., the terminals that transmit in present frame. If $\alpha_{i j}=0$, terminal $i$ does not transmit in slot $j$. During time slot $j, R$ receives the sum of the bursts with $\alpha_{i j} \neq 0$. From the received signal, $R$ tries to obtain the bit-wise XOR of the encoded messages as described in Section $\Pi$. The XOR is interpreted by $R$ as a linear equation in $G F\left(2^{n}\right)$, the coefficients of which are derived through the orthogonal preamble as described in Section IV If $N^{t x}$ 
TABLE I

EXAMPLE OF ACCESS PATTERN FOR THREE NODES TRANSMITTING IN A FRAME WITH $S=4$ SLOTS PER FRAME. $\alpha_{i j} \in G F\left(2^{n}\right)$ IS THE COEFFICIENT USED B Y NODE $i$ IN SLOT $j$. EACH COEFFICIENT CAN ASSUME ONE OF $q=2^{n}$ POSSIBLE VALUES, INCLUDING VALUE 0, WHICH CORRESPONDS TO THE CASE IN WHICH THE TERMINAL DOES NOT TRANSMIT.

\begin{tabular}{ccccc}
\hline & Slot 1 & Slot 2 & Slot 3 & Slot 4 \\
\hline$T_{1}$ & $\alpha_{11}$ & $\alpha_{12}$ & $\alpha_{13}$ & $\alpha_{14}$ \\
\hline$T_{2}$ & $\alpha_{21}$ & $\alpha_{22}$ & $\alpha_{23}$ & $\alpha_{24}$ \\
\hline$T_{3}$ & $\alpha_{31}$ & $\alpha_{32}$ & $\alpha_{33}$ & $\alpha_{34}$ \\
\hline
\end{tabular}

is the number of active terminals in a frame and assuming that all the received signals are decoded correctly, a linear system of equations in $G F\left(2^{n}\right)$ is obtained with $S$ equations and $N^{t x}$ variables. Each variable corresponds to a different source message. If $\mathbf{A}$ has rank equal to $N^{t x}$, then all the messages can be obtained by $R$. A necessary condition for $\mathbf{A}$ to be full rank is $N^{t x} \leq S$, i.e., the number of active terminals in a frame must be lower than the number of slots in a frame. Assuming Poisson arrivals with aggregate intensity $G$, the probability of such event is:

$$
\operatorname{Pr}\left\{N^{t x} \leq S\right\}=\sum_{n=0}^{S} \frac{(G S)^{n} e^{-G S}}{n !},
$$

which includes also the case in which there are no active terminals during a frame. For instance, in case of $S=100$ slots and $G=0.8$ the probability expressed by (6) is on the order of 0.99 . Even if $N^{t x}<S$, however, it can still happen that $\mathbf{A}$ is not full rank, i.e., not all the messages can be recovered. The probability that $\mathbf{A}$ is full rank for a given $N^{t x}<S$ depends on the MAC policy, and particularly on the probability distribution used to choose the coefficients.

One possibility is to use a uniform distribution for the coefficients (i.e., each coefficient can assume any value in $\left\{0, \ldots, 2^{n}-1\right\}$ with probability $\left.2^{-n}\right)$. In this case the number $d$ of transmitted replicas is a random variable, and the probability that $\mathbf{A}$ is full rank is [18]:

$$
P\left(S, N_{t x}\right)=\prod_{k=0}^{N_{t x}-1}\left(1-\frac{1}{2^{n(S-k)}}\right) .
$$

Using (6) and (7) we find the expression for the normalized throughput:

$$
\begin{aligned}
\Phi & =\frac{1}{S} \sum_{m=1}^{S} m \frac{(G S)^{m} e^{-G S}}{m !} P(S, m) \\
& =\frac{1}{S} \sum_{m=1}^{S} \frac{(G S)^{m} e^{-G S}}{(m-1) !} \prod_{k=0}^{m-1}\left(1-\frac{1}{2^{n(S-k)}}\right) \\
& =G \sum_{m=0}^{S-1} \frac{(G S)^{m} e^{-G S}}{m !} \prod_{k=0}^{m}\left(1-\frac{1}{2^{n(S-k)}}\right) .
\end{aligned}
$$


From Eqn. (8) we can see that $\Phi$ grows with $n$, which means that the system throughput increases with the size of the considered finite field. Moreover, we have:

$$
\begin{aligned}
\lim _{n \rightarrow \infty} \Phi & =\lim _{n \rightarrow \infty}\left[G \sum_{m=0}^{S-1} \frac{(G S)^{m} e^{-G S}}{m !} \prod_{k=0}^{m}\left(1-\frac{1}{2^{n(S-k)}}\right)\right] \\
& =G \sum_{m=0}^{S-1} \frac{(G S)^{m} e^{-G S}}{m !} .
\end{aligned}
$$

From Eqn. (9) it can be seen that the normalized throughput $\Phi$ tends to the probability of having less than $S$ transmitters in a frame as $n \rightarrow \infty$.

The MAC scheme we just analyzed presents one main drawback in terms of the energy efficiency of the protocol. As a matter of fact, given the frame length $S$, a node transmits each message on average $E[d]=S \times p$ times, $p=\left(1-2^{-n}\right)$ being the probability to choose a non-zero coefficient, i.e., the average number of transmissions grows linearly with $S$. In order to decrease the energy consumption, the probability of choosing the zero coefficient may be increased. However, a reduction in the transmission probability $p$ may affect the system throughput. In order to understand the relationship between the probability $p$ and the throughput $\Phi$, we refer to some results in random matrix theory. The problem can be formulated as follows: consider an $N^{t x} \times S$ random matrix $\mathbf{A}$ over $G F\left(2^{n}\right)$ with i.i.d. entries, each of which assumes value 0 with probability $p$ while with probability $1-p$ it assumes values in $\left\{1, \ldots, 2^{n}-1\right\}$. We are interested in the relationship between $p$ and the probability that $\mathbf{A}$ is full rank. In [19] the authors show that, if we want to achieve a rank $N^{t x}-O(1)$ with high probability, then, for $N^{t x}$ large, $p$ cannot be lower than $\frac{\log \left(N^{t x}\right)}{N^{t x}}$. At high loads (i.e., $G \simeq 1$ ), on average $N^{t x} \simeq S$, which means that, setting $p=\frac{\log (S)}{S}$, the average number of transmissions (and so the energy consumption) for each node is $E[d]=\log (S)$, i.e., it grows logarithmically with the number of slots in a frame. On the other side, $S$ must be kept large enough, as this increases the decoding probability, which makes the choice of small $S$ unpractical. With reference to the example considered earlier in this section, in which $S=100$, the average number of transmissions corresponding to the minimum required $p$ is equal to about 4.6. We evaluated numerically the effect a reduction of $p$ has on $\Phi$ for the case $S=100$ and $q=2^{8}$. We considered three cases. In the first one the transmission probability in each slot has been set to $p=1-2^{-n}=0.9961$, which corresponds to the case studied in the first part of this section and for which the throughput is given by Eqn. (8). In the second case we set $p$ just above the threshold, i.e., $p=0.0625>\frac{\log (S)}{S}=0.0461$, while in the last case $p$ has been set exactly equal to the threshold probability. Fig. 4 shows the results together with the numerical validation of Eqn. (8). It is interesting to note how passing from $p=0.9961$ to $p=0.0628$, with a reduction in transmission probability (or, equivalently, in average energy per message) of about $93.7 \%$, leaves the throughput unchanged, while a further decrease of $p$ of just another $1.5 \%$ leads to a $10 \%$ reduction in the maximum throughput with respect to the case $p=0.9961$. 


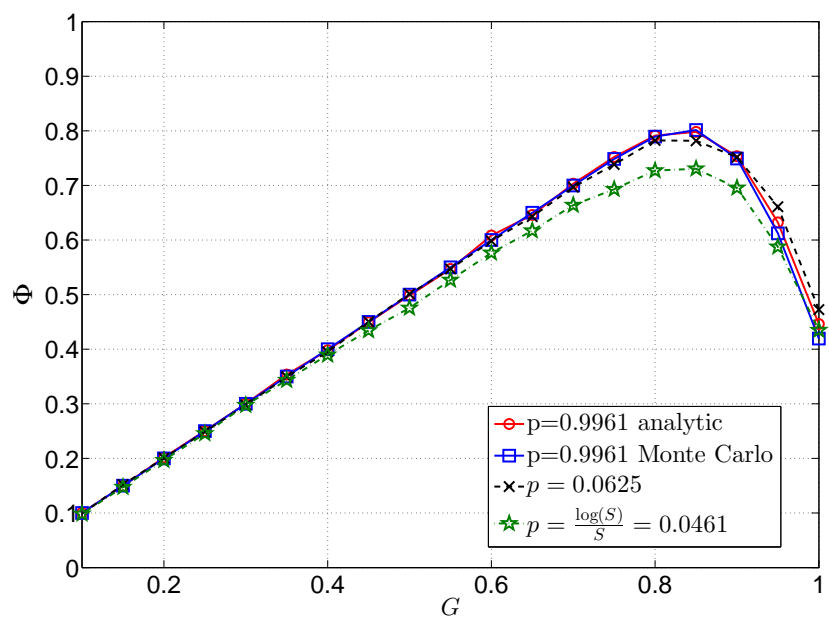

Fig. 4. Normalized throughput plotted against the normalized offered load for different values of the transmission probability $p$. We set $S=100$ slots per frame while the coefficients were chosen in $G F\left(2^{8}\right)$.

To further lower the energy consumption and control the number of repetitions $d$ (which, being a Bernoulli random variable, can theoretically assume values as large as $S$ ), an alternative is to fix the number of transmitted replicas a priori. Although this solution may lead in some cases to the impossibility of decoding all the transmitted messages, it may still be possible to recover many of them by using Gaussian elimination.

\section{IMPLEMENTATION ASPECTS}

\section{A. Channel Estimation and Node Identification}

For each frame the receiver $R$ needs to know which of the active terminals is transmitting in each slot and must have channel state information for each of the users. Both needs are addressed including an orthogonal preamble, such as the spreading codes used in CDMA, at the beginning of the burst. The use of an orthogonal preamble was proposed in [2] for the estimation of the phase in collided bursts. In [2] frequency offset and channel amplitude are derived from the clean bursts (i.e., bursts that did not experience collisions) and assumed to remain constant over the whole frame. Unlike in [2], the method we propose does not rely only on clean bursts. Thus the frequency offset and the amplitude of each transmitter must be estimated using the collided bursts for each frame. Although the performances of the estimator are likely to degrade with respect to the clean burst case, especially in case of high order collisions, the estimation can leverage in the information of all the collided bursts, which improves the estimation. For instance, if a packet is transmitted twice during a given frame and experiences collisions 
of order 2 in the first transmission and 4 in the second, the two estimations can be combined to obtain a better estimation of amplitude and frequency offset, which are constant during the whole frame.

In order to prove the feasibility of channel estimation in such conditions we show the results we obtained using the Estimate Maximize (EM) algorithm. We adopted the approach described in [20], where the EM algorithm is used to estimate parameters from superimposed signals. In [20] two examples were proposed related to multipath delay estimation and direction of arrival estimation. We apply the same approach to estimate amplitudes, phases and frequency offsets from the baseband samples of the received signal in case of a collision of size $k$. The algorithm is divided into an $E$ step, in which each signal is estimated, and an $M$ step, in which the mean square error between the estimation made at the $E$ step of current iteration and the signal reconstructed using parameters calculated in previous iteration is minimized with respect to the parameters to estimate. Formally, once initialized the parameters with randomly chosen values, at each iteration we have the following two steps:

Estimation step - for $i=1, \ldots, k$ calculate

$$
\begin{aligned}
\hat{p}_{i}^{(n)}(t) & =b_{i}(t) \hat{A}_{i}^{(n)} e^{j\left(2 \pi \widehat{\Delta \nu}_{i}^{(n)} T_{s} t+\hat{\varphi}_{i}^{(n)}\right)} \\
+ & \beta_{i}\left[r(t)-\sum_{l=1}^{k} b_{l}(t) \hat{A}_{l}^{(n)} e^{j\left(2 \pi \widehat{\Delta \nu}_{l}^{(n)} T_{s} t+\hat{\varphi}_{l}^{(n)}\right)}\right],
\end{aligned}
$$

Maximization step - for $i=1, \ldots, k$ calculate

$$
\min _{A^{\prime}, \Delta \nu^{\prime}, \varphi^{\prime}} \sum_{t=1}^{N^{p r e}}\left|b_{i}(t) \hat{p}_{i}^{(n)}(t)-A^{\prime} e^{j\left(2 \pi \Delta \nu^{\prime} T_{s} t+\varphi^{\prime}\right)}\right|^{2},
$$

where $p_{i}(t)$ is the preamble of burst $i$ after the matched filter, $A^{\prime}, \Delta \nu^{\prime}$ and $\varphi^{\prime}$ are tentative values for the parameters to be estimated, $N^{\text {pre }}$ is the preamble length, $b_{i}(t) \in\{ \pm 1\}$ is the t-th symbol in the preamble of the $\mathrm{i}$-th node and $T_{s}$ is the sampling period, taken equal to the symbol rate. $\beta_{i}$ are free parameters that we arbitrarily set to $\beta_{i}=0.8$, for $i=1, \ldots, k$.

We evaluated numerically the performance of the EM estimator assuming that phase offsets are uniformly distributed in $[-\pi,+\pi]$, frequency offsets are uniformly distributed in $\left[0, \Delta \nu^{\max }\right]$ with $\Delta \nu^{\max }$ equal to $1 \%$ of the symbol rate on the channel $\left(1 / T_{s}\right)$, and amplitudes are log-normally distributed. Figures 5, 6 and 7 show the mean squared error (MSE) of the estimation error for frequency, phase and amplitude, respectively. Amplitude error is normalized to the actual amplitude value while phase error is normalized to $\pi$. In the simulations we used as preambles Walsh-Hadamard words of length 128 symbols. The EM algorithm was run twice starting from randomly chosen initial values of the parameters and taking as result the values of the parameters that lead to the minimum of the sum across the signals of the error calculated in the last E step. This was done in order to reduce the probability 


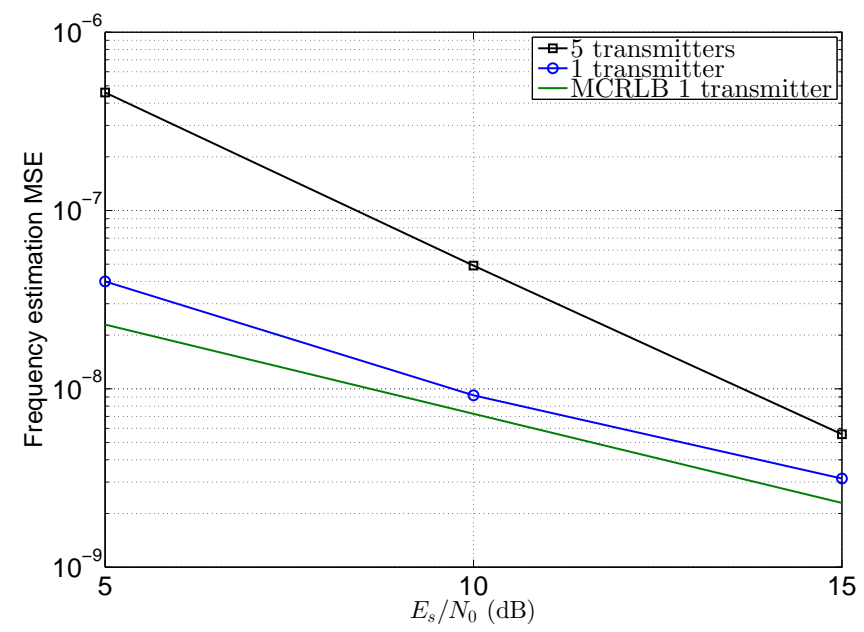

Fig. 5. Mean squared error (MSE) of the frequency offset estimation, i.e., $E\left[|\widehat{\Delta \nu}-\Delta \nu|^{2}\right]$. $E_{s}$ is the average energy per transmitted symbol for each node. The modified Cramer-Rao lower bound (MCRLB) for the case of one transmitter is also shown for comparison.

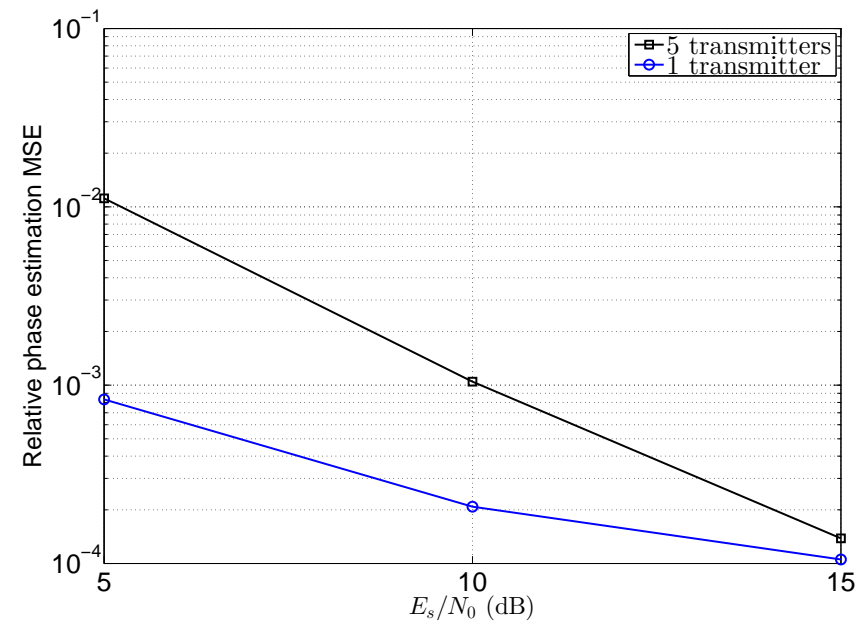

Fig. 6. MSE of the phase offset estimation normalized to $\pi$, i.e., $E\left[|\hat{\varphi}-\varphi|^{2}\right] / \pi^{2}$. $E_{s}$ is the average energy per transmitted symbol for each node.

to choose a "bad" local maximum, which is a problem that affects all the "hill climbing" algorithms. For each run 6 iterations were made.

In Fig. 8 the FER curves for different collision sizes obtained using the LLR values calculated in Section III are shown. The plots are obtained using a tail-biting duo-binary turbo code with rate $1 / 2$ and codeword length equal to 1504 symbols. The phase offsets $\varphi_{i}$ are random variables uniformly 


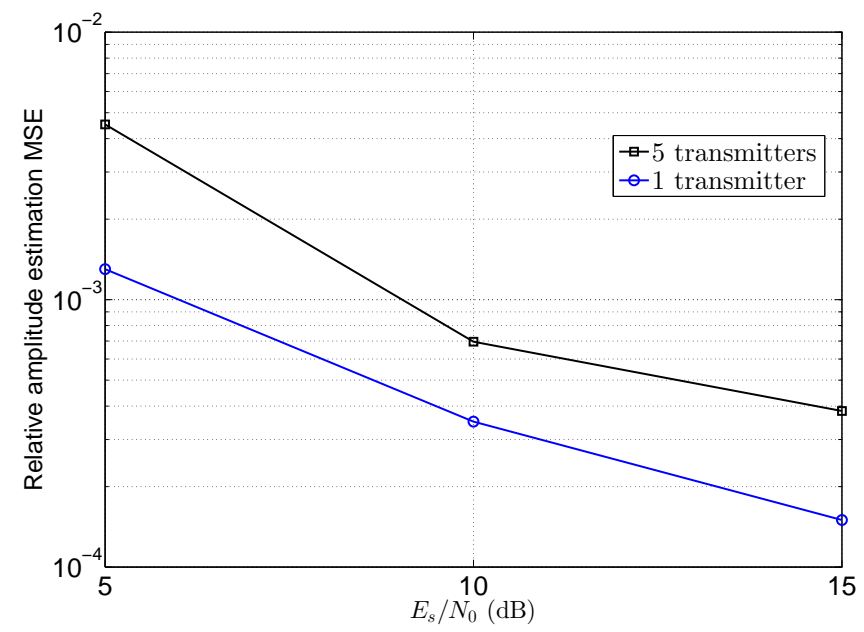

Fig. 7. MSE of the amplitude estimation normalized to the actual amplitude of the channel, i.e, $E\left[|\hat{A}-A|^{2} / A^{2}\right]$. $E_{s}$ is the average energy per transmitted symbol for each node.

distributed in $[-\pi,+\pi]$ while frequency offsets are uniformly distributed in $\left[0, \Delta \nu^{\max }\right]$ with $\Delta \nu^{\max }$ equal to $1 \%$ of the symbol rate $1 / T_{s}$. The FER curves for the case of estimated channels using the EM algorithm are also shown.

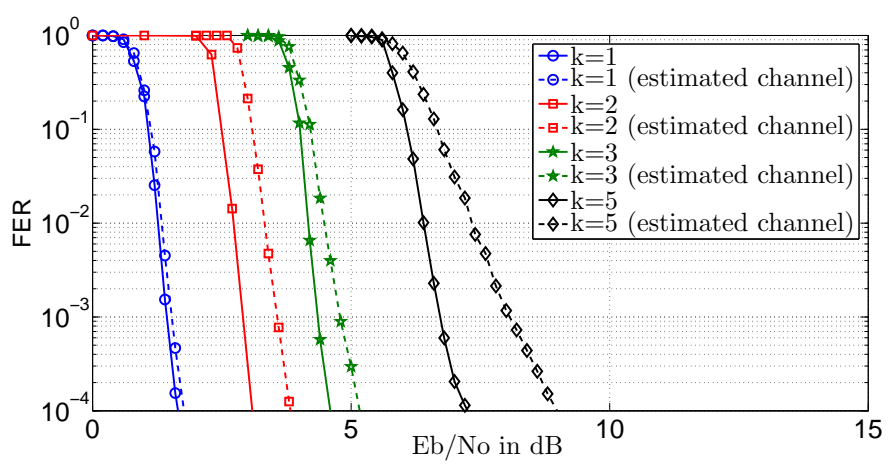

Fig. 8. FER for the XOR of transmitted messages for different numbers of transmitters. $E_{b}$ is the energy per information bit for each node. A tail-biting duo-binary turbo code with rate $1 / 2$ and codeword length 1504 symbols is used by all nodes. Phase offsets are uniformly distributed in $[-\pi,+\pi]$, frequency offsets are uniformly distributed in $\left[0, \Delta \nu^{\max }\right]$ with $\Delta \nu^{\max }$ equal to $1 \%$ of the symbol rate on the channel. Amplitudes are constant and equal to 1 . The FER curves for the case of estimated channels using the EM algorithm are also shown. 


\section{B. Error Detection}

An important issue in slotted ALOHA is the capability of the receiver to determine whether the received bursts are correctly decoded or not. This is particularly important in NCDP, where the error made in the decoding of a collision can propagate possibly leading to the loss of a whole frame. A common practice in packet networks is the use of a cyclic redundancy check (CRC), which allows to detect a wrong decoding with a certain probability. Some CRC's are based on a field which is appended to the message before channel coding, called CRC field. As the CRC operations are done in $G F(2)$ and by the linearity of the channel encoder, the CRC field in the message obtained by decoding a collision of size $k$ is a good CRC for $\mathbf{u}_{s}$, which is the bitwise XOR of the messages encoded in the $k$ collided signals. This allows to detect decoding errors, within the limits of the CRC capabilities, also in collided bursts. The implementation aspect of what type of CRC should be used is out of scope of this paragraph.

\section{Performance of Multi User Physical Layer Network Coding with IMPERFECT SYMBOL SYNCHRONIZATION}

In Section $\Pi$ we assumed that signals from different receivers add up with symbol synchronism at the receiver in case of a collision. In Fig. 9 an example is shown of received signal and sampling instants in

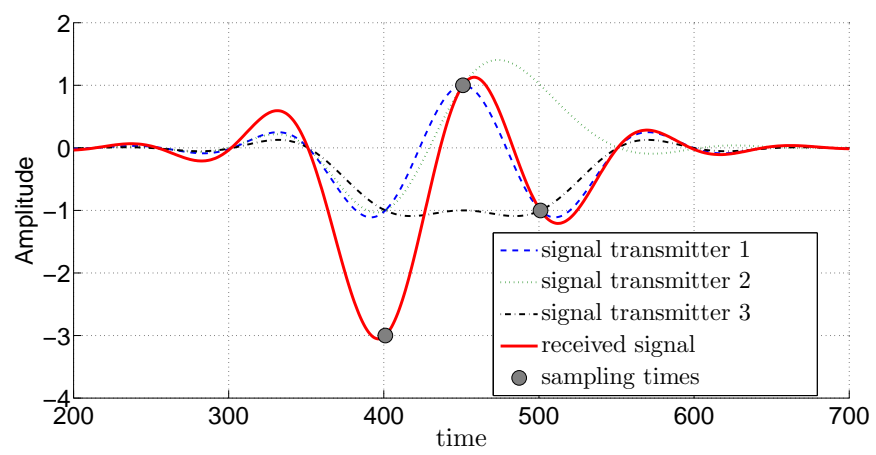

Fig. 9. Received signal after the matched filter in case of three colliding bursts with no timing offsets, i.e., $\Delta T_{1}=\Delta T_{2}=$ $\Delta T_{3}=0$. The transmitted signals after the matched filter in case of collision-free reception are also shown. The transmitted symbols are: [-1 $1-1],\left[\begin{array}{lll}-1 & 1 & 1\end{array}\right]$ and [-1 $\left.-1-1\right]$ for transmitter 1, 2 and 3, respectively. For sake of clarity, frequency and phase offsets as well as channel amplitudes were not included in the plot and the signals were considered as real. The samples, shown with grey circles in the figure, are taken at instants corresponding to the optimal sampling instants for each of the signals as if they were received without experiencing collision.

the case of three nodes transmitting with no timing offsets. The transmitted signals, which are also shown, modulate the sets of symbols [-1 $11-1],\left[\begin{array}{lll}-1 & 1 & 1\end{array}\right]$ and [ $\left.-1-1 \quad-1\right]$. The situation depicted in the figure is an illustrative one, as in a real system both I and Q signal components are present, signals may have different 
amplitudes, phase and frequency offsets for each of the bursts and the signal is immersed in thermal noise. However, in a real system there will always be a certain symbol misalignment, which grows larger as the resources dedicated to the synchronization phase diminish (see, e.g., [21] and references therein for examples of synchronization algorithms). Being able to cope with non perfect symbol synchronism can bring important advantages, such as less stringent constraints on signal alignment, with consequent savings in terms of network resources needed for the synchronization. In this section we study the effect of non perfect symbol synchronization and propose possible countermeasures. Let us consider a slotted multiple access with $k$ nodes accessing the channel at the same time. We assume that each transmitter has its own phase and frequency offsets. We further assume that each burst falls completely within the boundaries of a time slot, i.e., no burst can fall between two consecutive time slots. Let us call $T^{\prime}$ the time at which the peak of the first symbol of the bursts that first arrives at $R$. We define the relative delay (RD) $\Delta T_{i}$ of node $i$ as the temporal distance between the peak value of the first pulse of burst $i$ and $T^{\prime}$. In other words, the burst which arrives first at the receiver is used as reference, i.e., has RD equal to 0 . We assume SRRC pulses with roll off factor $\alpha$ are used. We further assume that all RD's belong to the interval $\left[0, \Delta T^{\max }\right]$, with $0 \leq \Delta T^{\max } \leq T_{s} / 2$.

In case of a collision of $k$ bursts, the received signal before the matched filter is:

$$
y(t)=\sum_{i=1}^{k} s_{i}(t)+w(t),
$$

where,

$$
s_{i}(t)=A_{i} \sum_{l=1}^{N} b_{i}(l) g\left(t-l T_{s}-\Delta T_{i}\right) e^{j\left(2 \pi \Delta \nu_{i} t+\varphi_{i}\right)},
$$

$N$ being the number of symbols in the burst, $g(t)$ is the square root raised cosine pulse and $w(t)$ represents an AWGN process. The samples taken after the matched filter at times $t_{l}$ are:

$$
r\left(t_{l}\right)=\left.y(t) \otimes g(-t)\right|_{t=t_{l}}=\sum_{i=1}^{k} q_{i}\left(t_{l}\right)+n\left(t_{l}\right),
$$

where,

$$
q_{i}\left(t_{l}\right)=A_{i} \sum_{l=1}^{N} b_{i}(l) p\left(t_{l}-l T_{s}-\Delta T_{i}\right) e^{j\left(2 \pi \Delta \nu_{i} t_{l}+\varphi_{i}\right)},
$$

$p(t)$ being the raised cosine pulse, $\otimes$ is the convolution operator and $n(t)$ is the noise process after filtering and sampling. Note that in (15) the exponential term is treated as a constant. This approximation is done under the assumption that $\Delta \nu T_{s} \ll 1$, i.e., the exponential term is almost constant over many symbol cycles.

The sampled signal is then sent to the channel decoder. It is not clear at this point which is the optimal sampling time, as the optimal sampling time for each of the bursts taken singularly may be different. Moreover, sampling the signal just once may not be the optimal choice. Actually, as we will 
show in next section, the performance of the decoder is quite poor in case a single sample per symbol is taken.

In the following we propose several techniques to mitigate the impairment due to imperfect symbol synchronization. We assume that $R$ has knowledge of the relative delays of all the transmitters, which can be derived through the orthogonal preambles. We further assume that $R$ has perfect CSI for each of the transmitters. Without loss of generality and for ease of exposition, from now on we will refer to the sampling time for the symbol number 1 .

\section{A. Single sample}

a) Mean Delay: The first method we present is Mean Delay (MD). In MD the received signal is sampled just once per symbol. The sampling time is chosen to be the mean of the relative delay, i.e.:

$$
T^{M D}=\frac{1}{k} \sum_{m=1}^{k} \Delta T_{m} .
$$

The sample $r\left(T^{M D}\right)$ is then used to calculate the LLR's as in Eqn. (5). ISI is not taken into account.

\section{B. Multiple samples}

In the following we describe four different methods that use $k$ samples per symbol, $k$ being the collision size.

We start by describing two methods in which the symbol is sampled $k$ times in correspondence of the RD's. Due to the non perfect synchronization, when the signal is sampled in $\Delta T_{i}$ the sample obtained is the sum of the first symbol of each of the users, weighted by the relative channel coefficient, plus a term of ISI due to signals $s_{j}, j \in\{1, \ldots, k\}, j \neq i$, which are sampled at non ISI-free instants. As the LLR's need the channels of each of the users, the ISI should be taken into account. However, the ISI is a function of many (theoretically all) symbols, and can not be taken into account exactly. In Fig. 10 the received signal after the matched filter is shown in the case of three colliding bursts with timing offsets $\Delta T_{1}=0, \Delta T_{2}=T_{s} / 6$ and $\Delta T_{3}=T_{s} / 4$. The transmitted signals after the matched filter in the case of collision-free reception are also shown. The symbols transmitted by each terminal are the same as in Fig. 9 The samples, shown with grey circles in the figure, are taken in correspondence of the RD's, which coincide with the optimal sampling instants for each of the signals as if they were received without experiencing collision.

b) Mean LLR: In Mean LLR (ML) the received signal is sampled $k$ times in the instants correspondent to $\Delta T_{i}, i=1, \ldots, k$. For each of the samples the LLR's are calculated as in (5). Then the average of the $k$ LLR's is passed to the decoder. 


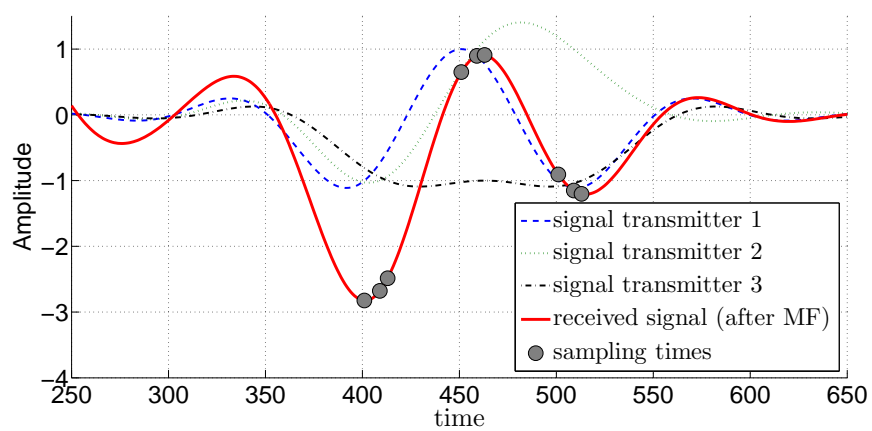

Fig. 10. Received signal after the matched filter in case of three colliding bursts with timing offsets $\Delta T_{1}=0, \Delta T_{2}=T_{s} / 6$ and $\Delta T_{3}=T_{s} / 4$. The transmitted signals after the matched filter in the case of collision-free reception are also shown. The transmitted symbols are: [- $\left.\begin{array}{lll}-1 & 1 & -1\end{array}\right],\left[\begin{array}{lll}-1 & 1 & 1\end{array}\right]$ and [-1 $\left.-1-1\right]$ for transmitter 1, 2 and 3, respectively. The samples, shown with grey circles in the figure, are taken at instants corresponding to the optimal sampling instants for each of the signals as if they were received without experiencing collision. Unlike in the case of perfect symbol alignment, here more than one sample per symbol is taken.

c) Mean Sample: As in ML, also in Mean Sample (MS) $r(t)$ is sampled $k$ times in correspondence of the relative delays. The difference between the two methods is that in MS the samples are averaged out to obtain the mean sample:

$$
\bar{r}(t)=\frac{1}{k} \sum_{m=1}^{k} r\left(\Delta T_{m}\right) .
$$

Finally, $\bar{r}(t)$ is used in the (5) instead of $r(t)$.

d) Uniform Sampling: In Uniform Sampling (US) the signal is sampled $k$ times as in previous methods, but the sampling times do not correspond to the RD's. The sampling times are chosen uniformly in $\left[0, \Delta T^{\max }\right]$, i.e, in case of $k$ transmitters the samples are taken at intervals of $\Delta T^{\max } /(k-1)$. Then, as in MS, the samples are averaged out and used in the calculation of the LLRs. This method has the advantage that receiver does not need the knowledge of the RD's in order to decode and the sampling itself is simplified as it is done uniformly in each symbol.

e) Equivalent Channel: The received signal is sampled $k$ times in the instants correspondent to $\Delta T_{i}, i=1, \ldots, k$. In the method Equivalent Channel (EC) the amplitude variation of the channel of each user due to imperfect timing is taken into account for the current symbol. Note that the ISI is not taken into account, but only the variation in amplitude of present symbol due to imperfect timing is accounted for. Assuming that the received signal is sampled at time $t=\Delta T_{i}$, then the channel coefficient of burst $q$ that is used in the LLR is:

$$
h_{q}^{e q}(t)=A_{q} e^{j\left(2 \pi \Delta \nu_{q} T_{s} \Delta T_{i}+\varphi_{q}\right)} p\left(\Delta T_{i}-\Delta T_{q}\right),
$$


$p(t)$ being the raised cosine pulse. After the sampling, the $k$ samples per symbol are averaged together and used in the LLR instead of $r(t)$. This sampling procedure is equivalent (apart from the ISI) to filtering the received signal using a filter which is matched not to the single pulse, but to the pulse resulting from the delayed sum of $M$ pulses. In Fig. 11 the frame error rate is shown for the case of 5 transmitters with delays uniformly distributed in $\left[0, T_{s} / 4\right]$. Constant channel amplitudes were considered, while phases and frequency offsets are i.i.d. random variables in $[0,2 \pi]$ and $\left[0, \Delta \nu_{\max }\right]$ respectively, where $\Delta \nu_{\max }$ is equal to $1 /\left(100 T_{s}\right)$. The results for the 5 different methods are shown together with the FER for the case of ideal symbol synchronism. The methods that use more than one sample per symbol perform significantly better than MD, which uses only one sample per symbol. Among the methods based on oversampling, MS and EC perform slightly better than the other two. The FER of all methods present a lower slope w.r.t. the ideal case. The loss is about $1 \mathrm{~dB}$ at $F E R=10^{-2}$ for the methods that use oversampling.

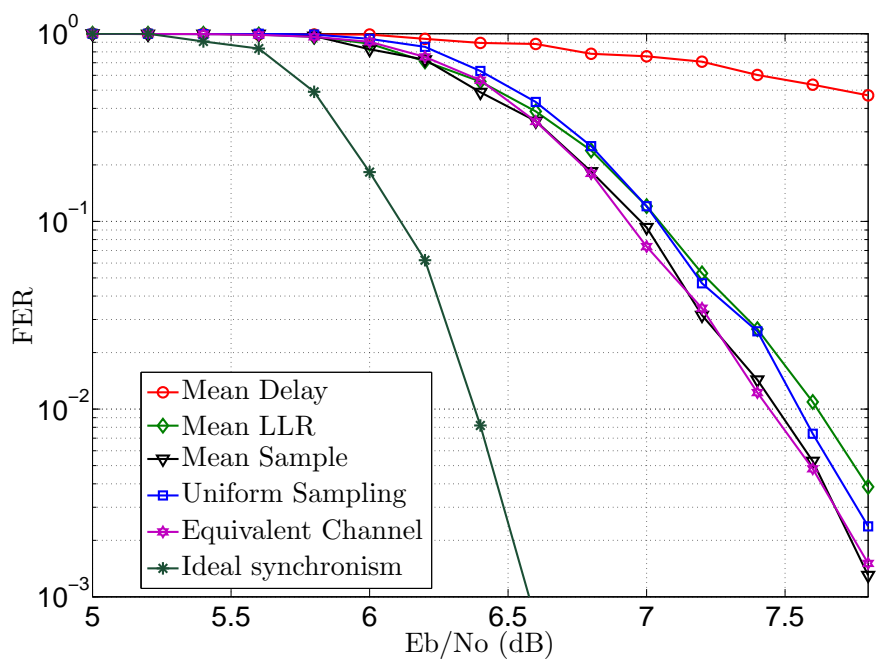

Fig. 11. Frame error rate for decoding a collision of size 5 with independent frequency and phase offsets across the transmitters and delays uniformly distributed in $\left[0, T_{s} / 4\right]$. A roll-off factor of $\alpha=0.35$ was used. The results for the 5 different methods are shown together with the FER for the case of ideal symbol synchronism. Oversampling significantly improves the FER with respect to the case of single sample. The two methods that exploit knowledge of relative delays, i.e, MS and EC, perform slightly better than the others. The FER of all methods present a lower slope w.r.t. the ideal case, losing about $1 \mathrm{~dB}$ at $F E R=10^{-2}$ for the methods that use more than one sample. 


\section{NUMERICAL RESULTS}

In this section we present the numerical results. Our performance metrics are the normalized throughput $\Phi$ defined as:

$$
\Phi=G(1-\Upsilon)
$$

where $\Upsilon \in[0,1]$ is the average packet loss rate (i.e, the ratio of the number of lost packets to the total number of packets that arrive at the transmitters), and the average energy consumption per received message $\eta$, defined as the average number of transmissions needed for a message to be correctly received by $R$. We consider two benchmarks. The first one is a system that implements the contention resolution diversity slotted ALOHA (CRDSA) protocol, which has been proposed in [2]. In CRDSA a node transmits two or more copies of a burst (twin bursts) in different slots randomly chosen within a frame. Each of the twin bursts contains information about the position of the other twin bursts in the frame. If one of the twin bursts does not experience a collision (i.e, it is clean) and can be correctly decoded, the position of the other twin bursts is known. These bursts may or may not experience a collision with other bursts. If it happens, these are removed through interference cancelation using the decoded bursts. In order to do this $R$ memorizes the whole frame, decodes the clean bursts, reconstructs the modulated signals and, once the effect of each user's channel has been included in the reconstruction, they are subtracted from the slots in which their replicas are located. The IC process is iterated for a number $N^{i t e r}$ of times, at each time decoding the bursts that appear to be "clean" after the previous IC iteration. The second benchmark is a slotted ALOHA system.

We consider two different setups. In one, the nodes do not receive any feedback by the receiver, while in the second setup $R$ gives some feedback to the active terminals. For this last case we consider an automatic repeat request (ARQ) scheme, in which a node receives an acknowledgement (ACK) or a negative acknowledgement (NACK) from the receiver in case a message is or is not correctly received, respectively. An alternative to the NACK is to having the transmitters using a counter for each transmitted packet, indicating the time elapsed since it has been transmitted. If the timer exceeds a threshold value (which depends on the system's RTT), the message is declared to be lost. A node that receives a NACK (or whose timer exceeds the threshold vale) enters a backlog state. Backlogged nodes retransmit the message for which they received the NACK in another frame, uniformly chosen at random among the next $B$ frames. We call $B$ the maximum backlog time. The process goes on until the message is acknowledged [22]. In both setups we assume a very large population of users. Furthermore, we assume that the average SNR is high enough so that the FER at the receiver is negligible.

In the first setup, in which no feedback is provided by the receiver, the average amount of energy spent by a node for each message which is correctly received does not change with the system load $G$, and is equal to the average number of times a message is repeated within a frame. In Fig. 12 the normalized throughput $\Phi$ is plotted against the normalized traffic load $G$. The normalized traffic load is 


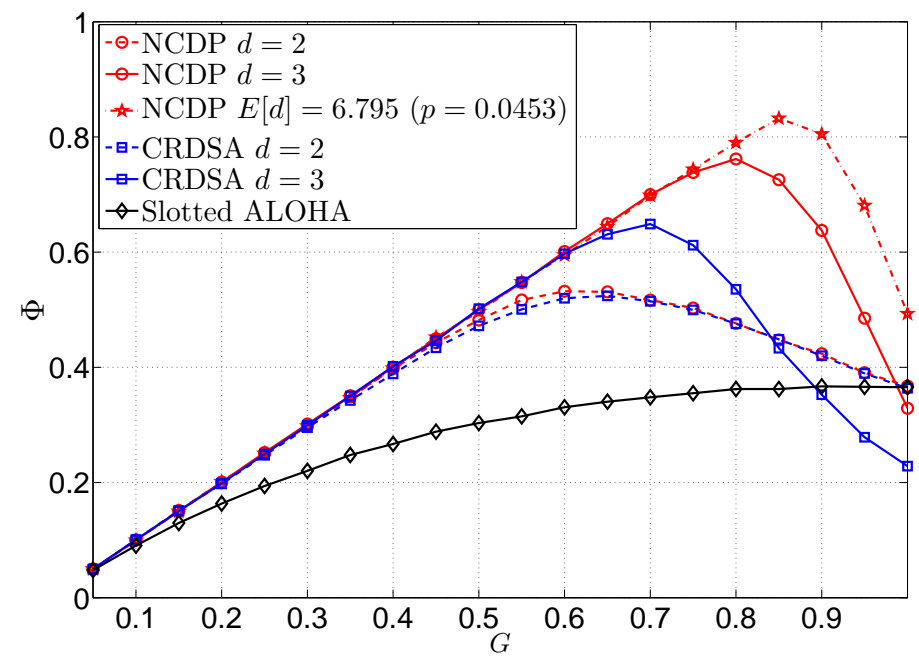

Fig. 12. Normalized throughput $\Phi$ vs normalized traffic load $G$. The normalized traffic load is the average rate at which new messages are injected in the network, and is independent from the number of times a message is repeated within a slot. In the simulation the frame size was set to $S=150$ slots. No feedback was assumed from the receiver.

the average rate at which the new messages (i.e, messages which are being transmitted for the first time) are injected in the network, and is independent from the number of times a message is repeated within a slot. In the figure, the throughput curves of NCDP and CRDSA schemes in case of $d=2$ and $d=3$ replicas are shown. The throughput curve for NCDP in case of a constant retransmission probability $p=0.0453$ is also shown. Note that this probability is above the threshold value we mentioned in Section V] as for $S=150$ we have $\log (S) / S=0.0334$. The scheme with $p=0.0453$ outperforms all the others in terms of throughput, achieving a peak value of about 0.8 . It is interesting to note how increasing the number of transmissions per message (and so the energy consumption) leads to an increase in the peak throughput of the system. However, $\Phi$ increases about 0.2 when passing from $d=2$ to $d=3$ repetitions, while the increase in the peak throughput is only about 0.05 when passing from $d=2$ repetitions per message to an average of $E[d]=6.795$ in case of a fixed transmission probability.

In the second setup, in which retransmissions are allowed, we evaluate jointly the spectral efficiency (average number of messages successfully received per slot) and the energy consumption (average number of transmissions needed for a message to be correctly received) of the schemes under study. In Fig. 13, $\Phi$ is plotted against $G$ for a frame size $S=150$ slots and a maximum backlog time $B=50$ frames. The figure shows how $\Phi$ increases linearly with $G$ up to a threshold load value. Such threshold increases with the (average) number of repetitions of the considered scheme. The $\Phi$ curve of NCDP upperbounds that of CRDSA. The reason for this lies in the way the decoding process is carried out 
by the receiver $R$ in NCDP. $R$ first tries to decode the whole frame, which is feasible if the coefficient matrix $\mathbf{A}$ has rank $N^{t x}$. If the whole frame can not be decoded, then $R$ applies Gaussian elimination on $\mathbf{A}$, in order to recover as many messages as possible. It can be easily verified that Gaussian elimination in NCDP is the equivalent, in a finite field, of the IC process of CRDSA, which is applied in the analog domain. In order to compare jointly the spectral and the energy efficiency of the different schemes,

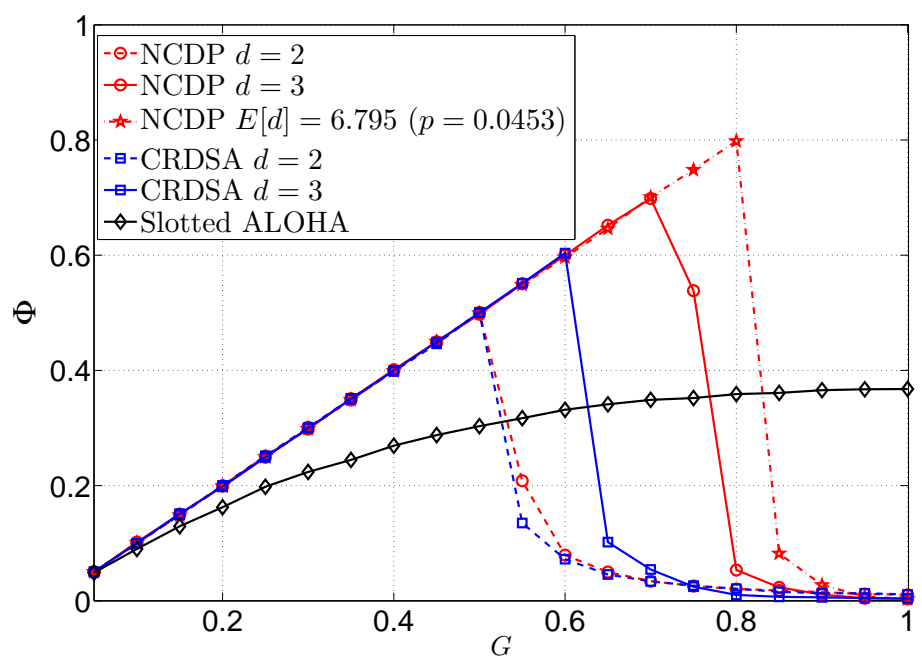

Fig. 13. Normalized throughput $\Phi$ vs normalized traffic load $G$ in a system with retransmission. In the simulation the frame size was set to $S=150$ slots while the maximum backlog time was set to $B=50$ frames.

we plot the curves for the normalized throughput vs the average energy consumption per received message $\eta$, which is shown in Fig. 14. The increase in throughput coming from an increased number of transmissions implies a higher energy consumption for a given transmitter in a given frame. However, this does not necessarily implies a loss in energy efficiency. As a matter of facts, the simulation results we are going to present show that there is not a scheme that outperforms the others in terms of both energy and spectral efficiency, but which scheme is best depends on the maximum throughput we want to achieve. In Fig. 14 we see that SA achieves a higher throughput with a lower energy consumption with respect to the other schemes in the region $\Phi<0.35$. In the region $\Phi>0.35$, instead, both NCDP and CRDSA achieve a higher throughput with lower energy consumption with respect to SA. NCDP and CRDSA behave almost in the same way in the case of 2 repetitions, achieving a maximum throughput of 0.5 for an average energy consumption of 2 . In the case of 3 repetitions NCDP achieves a maximum $\Phi$ of 0.7 , higher than CRDSA, for which the peak value is 0.6 , for $\eta=3$. In the NCDP scheme with a retransmission probability of $p=0.0453$ a peak throughput of 0.8 is achieved in correspondence of an average energy consumption of $\eta=6.795$. For comparison, we also show the throughput-energy 


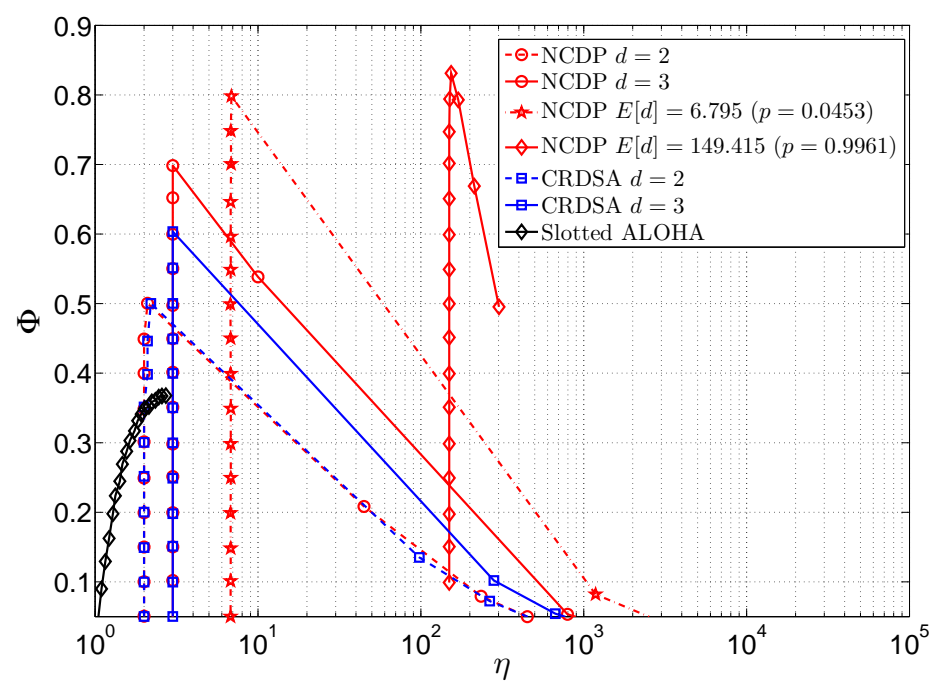

Fig. 14. Normalized throughput vs average energy consumption per decoded message for $S=150$ and $B=50$ frames.

curve for NCDP in case of $p=0.9961$, i.e., coefficients $\alpha$ are chosen uniformly in $G F\left(2^{8}\right)$. The high $p$ leads to a high throughput, but also to a high energy consumption, with a minimum of $\eta=149.415$. Moreover, we note that the gain with respect to the scheme with $p=0.0453$ is negligible (about $5 \%$ ), especially when compared to the energy saving of about $95 \%$ of this last one.

\section{Conclusions}

We have proposed a new collision recovery scheme for symbol-synchronous slotted ALOHA systems based on PHY layer NC over extended Galois Fields. This allows to better exploit the diversity of the system, leading to increased spectral efficiency and, depending on the system load, to an increased energy efficiency. We have compared the proposed scheme with two benchmark schemes in two different setups. One is a best-effort setup, in which the nodes do not receive any feedback from the receiver. In the second setup feedback is allowed from the receiver and an ARQ mechanism is assumed. In the second setup we have evaluated jointly the spectral efficiency and the energy consumption of the proposed scheme and compared it with other collision resolution schemes previously proposed in the literature. Once the PHY layer NC is applied to decode the collided bursts, the receiver applies common matrix manipulation techniques over finite fields, which results in a high-throughput scheme. The increase in throughput coming from an increased number of transmissions implies a higher energy consumption for a given transmitter in a given frame. However, this does not necessarily implies a loss in energy efficiency. We showed that NCDP achieves a higher spectral efficiency with respect to the considered benchmarks, while there is not a single scheme that outperforms the others in terms of both energy and 
spectral efficiency, but the best scheme depends on the maximum achievable throughput.

Furthermore, we carried out an analysis of several physical layer issues related to multi-user PHY NC. We extended the analysis on and proposed countermeasures against the effects of physical layer impairments on the FER when applying PHY NC for a generic number of colliding signals. In particular, we took into account frequency and phase offsets at the transmitters which, up to our knowledge, have been previously addressed only for the case of two colliding signals. Finally, we showed the feasibility of channel estimation for PHY NC in the presence of more than two colliding signals and studied the effect of non perfect symbol synchronism on the decoder FER, proposing four different methods to compensate for such effect. Up to our knowledge, this kind of analysis has been carried out only for the case of two colliding signals and mainly in the context of two-way relay communication.

\section{APPENDIX}

Starting from the samples $r\left(t_{l}\right)$ the receiver $R$ wants to decode the codeword $\mathbf{x}_{s} \triangleq \mathbf{x}_{1} \oplus \mathbf{x}_{2} \oplus \ldots \oplus \mathbf{x}_{k}$, where $\oplus$ denotes the bit-wise XOR. In order to do this we must feed the decoder of $R$ with the vector $\mathbf{L}^{\oplus}=\left\{L^{\oplus}(1), \ldots, L^{\oplus}(N)\right\}$ of LLRs for $\mathbf{x}_{s}$. We have:

$$
\begin{aligned}
L^{\oplus}(l) & \triangleq \ln \left\{\frac{\operatorname{Pr}\left[x_{s}(l)=1 \mid r\left(t_{l}\right)\right]}{\operatorname{Pr}\left[x_{s}(l)=0 \mid r\left(t_{l}\right)\right]}\right\} \\
& =\ln \left\{\frac{\operatorname{Pr}\left[r\left(t_{l}\right) \mid x_{s}(l)=1\right]}{\operatorname{Pr}\left[r\left(t_{l}\right) \mid x_{s}(l)=0\right]}\right\} .
\end{aligned}
$$

The last equality follows from the symmetry of the XOR operator provided that $x_{j}(l)$ 's are independent and identically distributes (i.i.d.) with $\operatorname{Pr}\left[x_{j}(l)=1\right]=\operatorname{Pr}\left[x_{j}(l)=0\right]=\frac{1}{2}$. Equation (20) reduces to the calculation of the ratio of the likelihood functions of $r\left(t_{l}\right)$ for the cases $x_{s}(l)=1$ and $x_{s}(l)=0$. We indicate these functions as $f_{1}\left(r\left(t_{l}\right)\right)$ and $f_{0}\left(r\left(t_{l}\right)\right)$ respectively. Functions $f_{0}\left(r\left(t_{l}\right)\right)$ and $f_{1}\left(r\left(t_{l}\right)\right)$ are Gaussian mixtures:

$$
f_{1}\left(r\left(t_{l}\right)\right)=\frac{2^{-k}}{\sqrt{2 \pi N_{0}}} \sum_{i=1}^{\left\lfloor\frac{k+1}{2}\right\rfloor} \sum_{m=1}^{\left(\begin{array}{c}
k \\
2 i-1
\end{array}\right)} e^{-\frac{\left|r\left(t_{l}\right)-\mathbf{d}^{o}(2 i-1, m)^{T} \mathbf{h}\left(t_{l}\right)\right|^{2}}{2 N_{0}}}
$$

$\mathbf{h}\left(t_{l}\right)$ being a column vector containing the channel coefficients of the $k$ transmitters at time $t_{l}$ (which change at each sample due to frequency offsets), while $\mathbf{d}^{o}(2 i-1, m)$ is a column vector containing one (the m-th) of the $\left(\begin{array}{c}k \\ 2 i-1\end{array}\right)$ possible permutations over $k$ symbols (without repetitions) of an odd number $(2 i-1)$ of symbols with value " +1 ". As for the case with $x_{s}=0$ we have:

$$
f_{0}\left(r\left(t_{l}\right)\right)=\frac{2^{-k}}{\sqrt{2 \pi N_{0}}} \sum_{i=1}^{\left\lfloor\frac{k+1}{2}\right\rfloor} \sum_{m=1}^{\left(\begin{array}{c}
k \\
2 i
\end{array}\right)} e^{-\frac{\left|r\left(t_{l}\right)-\mathbf{d}^{e}(2 i, m)^{T} \mathbf{h}\left(t_{l}\right)\right|^{2}}{2 N_{0}}},
$$

where $\mathbf{d}^{e}(2 i, m)$ is a column vector containing one (the m-th) of the $\left(\begin{array}{c}k \\ 2 i\end{array}\right)$ possible permutations over $k$ symbols (without repetitions) of an even number ( $2 i$ ) of symbols with value " +1 ”. Finally using (21) 
and (22) in (20) we find the following expression for the LLR:

$$
L^{\oplus}(l)=\ln \left\{\frac{\sum_{i=1}^{\left\lfloor\frac{k+1}{2}\right\rfloor} \sum_{m=1}^{\left(\begin{array}{c}
k \\
2 i-1
\end{array}\right)} e^{-\frac{\left|r\left(t_{l}\right)-\mathbf{d}^{o}(2 i-1, m)^{T} \mathbf{h}\left(t_{l}\right)\right|^{2}}{2 N_{0}}}}{\sum_{i=1}^{\left\lfloor\frac{k+1}{2}\right\rfloor} \sum_{m=1}^{\left(\begin{array}{c}
k \\
2 i
\end{array}\right)} e^{-\frac{\left.\mid r\left(t_{l}\right)-\mathbf{d}^{e}(2 i, m)\right)\left.^{T} \mathbf{h}\left(t_{l}\right)\right|^{2}}{2 N_{0}}}}\right\} .
$$

\section{REFERENCES}

[1] G. Choudhury and S. Rappaport, "Diversity ALOHA - a random access scheme for satellite communications," IEEE Trans. on Comm., vol. 31, no. 3, pp. 450-457, Mar. 1983.

[2] E. Casini, R. De Gaudenzi, and O. d. R. Herrero, "Contention resolution diversity slotted ALOHA (CRDSA): An enhanced random access scheme for satellite access packet networks," IEEE Trans. on Wireless Comm., vol. 6, no. 4, pp. 1408-1419, Apr. 2007.

[3] H. C. Bui, J. Lacan, and M.-L. Boucheret, "NCSA: A new protocol for random multiple access based on physical layer network coding," http://arxiv.org/abs/1009.4773, 2010.

[4] G. Liva, "Graph-based analysis and optimization of contention resolution diversity slotted ALOHA," IEEE Trans. on Comm., vol. 59, no. 2, pp. 477-487, Feb. 2011.

[5] S. Zhang, S. Liew, and P. Lam, "Physical layer network coding," in ACM MOBICOM, Los Angeles (CA), U.S.A., Sep. 2006.

[6] F. Rossetto and M. Zorzi, "On the design of practical asynchronous physical layer network coding," in IEEE Workshop on Signal Proc. Advances in Wireless Comm., Perugia, Italy, June 2009.

[7] R. H. Y. Louie, Y. Li, and B. Vucetic, "Practical physical layer network coding for two-way relay channels: Performance analysis and comparison," IEEE Trans. on Wireless Comm., vol. 9, no. 2, pp. 764-777, Feb. 2010.

[8] J. H. Sorensen, R. Krigslund, P. Popovski, T. Akino, and T. Larsen, "Physical layer network coding for FSK systems," IEEE Comm. Letters, vol. 13, no. 8, Aug. 2009.

[9] F. Rossetto, "A comparison of different physical layer network coding techniques for the satellite environment," in Advanced Satellite Multimedia Systems Conf. (ASMS), Cagliari, Italy, Sep. 2010.

[10] M. Durvy, C. Fragouli, and P. Thiran, "Towards reliable broadcasting using ACKs," in IEEE Int'l Symp. on Info. Theo. (ISIT), Nice, France, June 2007.

[11] C. H. Foh, J. Cai, and J. Qureshi, "Collision codes: Decoding superimposed BPSK modulated wireless transmissions," in IEEE Consumer Comm. and Networking Conf., Las Vegas (NV), U.S.A., Jan. 2010.

[12] B. Nazer and M. Gastpar, "Reliable physical layer network coding," Proceedings of the IEEE, vol. 99, no. 3, pp. 438-460, Mar. 2011.

[13] G. Cocco, C. Ibars, D. Gündüz, and O. d. R. Herrero, "Collision resolution in slotted ALOHA with multi-user physical-layer network coding," in IEEE Vehicular Technology Conf. (VTC Spring), Budapest, Hungary, May 2011.

[14] D. Maduike, H. D. Pfister, and A. Sprintson, "Design and implementation of physical-layer network-coding protocols," in Asilomar Conference on Signals, Systems and Computers, Pacific Grove (CA), U.S.A., Nov. 2009. 
[15] L. Lu and S. C. Liew, “Asynchronous physical-layer network coding," IEEE Trans. on Wireless Comm., vol. 11, no. 2, pp. 819-831, Feb. 2012.

[16] M. Jain, S. L. Miller, and A. Sprintson, "Parameter estimation and tracking in physical layer network coding," in IEEE Global Telecomm. Conf., Houston (TX), U.S.A., Dec. 2011.

[17] R. Koetter and M. Medard, “An algebraic approach to network coding," in IEEE Int'l Symp. on Info. Theo., Washington, D.C., U.S.A., June 2001.

[18] O. Trullols-Cruces, J. M. Barcelo-Ordinas, and M. Fiore, "Exact decoding probability under random linear network-coding," IEEE Comm. Letters, vol. 15, no. 1, pp. 67-69, Jan. 2011.

[19] J. Blömer, R. Karp, and E. Welzl, "The rank of sparce random matrices over finite fiels," Random Structures and Algorithms, vol. 10, no. 4, pp. 407-419, July 1997.

[20] M. Feder and E. Weinstein, "Parameter estimation of superimposed signals using the EM algorithm," IEEE Trans. on Acoustics, Speech and Signal Processing, vol. 36, no. 4, pp. 477-489, Apr. 1988.

[21] R. D. J. Van Nee, “Timing aspects of synchronous CDMA," in IEEE Int'l Symp. on Personal, Indoor and Mobile Radio Comm., The Hague, The Netherlands, Sep. 1994.

[22] L. Kleinrock and S. Lam, "Packet switching in a multiaccess broadcast channel: Performance evaluation," IEEE Trans. on Comm., vol. 23, no. 4, pp. 410-423, Apr 1975. 